Supporting Information

\title{
The Impact of Solvent Quality on the Heck Reaction: Detection of Hydroperoxide in NMP
}

Qiaogong Su* and Hayao Matsuhashi ${ }^{*}$

Chemical Development, GlaxoSmithKline, 1250 South Collegeville Road, Collegeville, Pennsylvania

19426, United States

\section{Table of Contents}

1. Copies of ${ }^{1} \mathrm{H}$ and ${ }^{13} \mathrm{C}$ NMR spectra for Compound 1................................................. S2

2. ${ }^{1} \mathrm{H}$ NMR monitoring of NMP-5-OOH generated by bubbling air through NMP at room

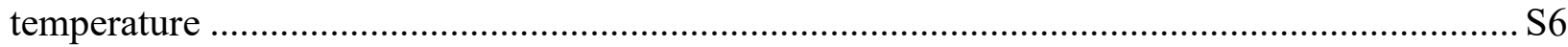

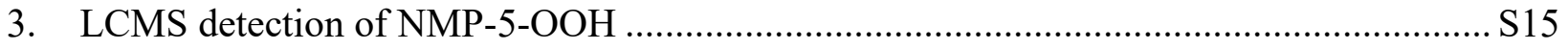


1. Copies of ${ }^{1} \mathrm{H}$ and ${ }^{13} \mathrm{C}$ NMR spectra for Compound 1

$$
\text { water| }
$$$$
\begin{array}{lr}
\text { carbon } & \\
J_{F, 4}= & 5.0 \\
J_{F, 4}= & 9.1 \\
J_{F, s}= & 4.0 \\
J_{F, s}= & 8.0 \\
J_{F, T}= & 14.1 \\
J_{F, T}=250.5 \\
J_{F, s}=11.1 \\
J_{F, s=}=244.5 \\
J_{F, s}=18.1
\end{array}
$$

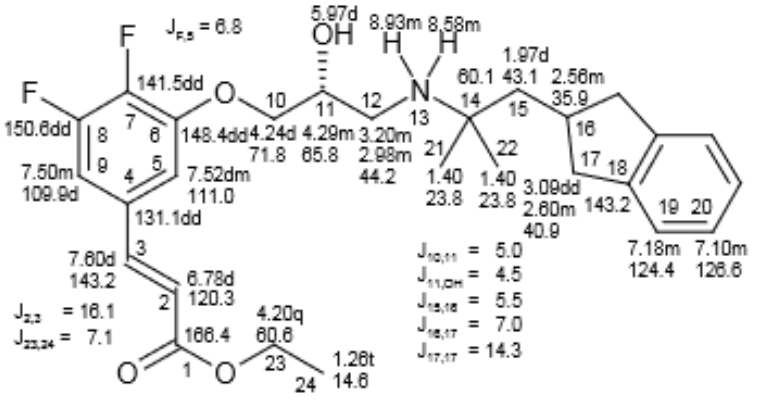

(1)

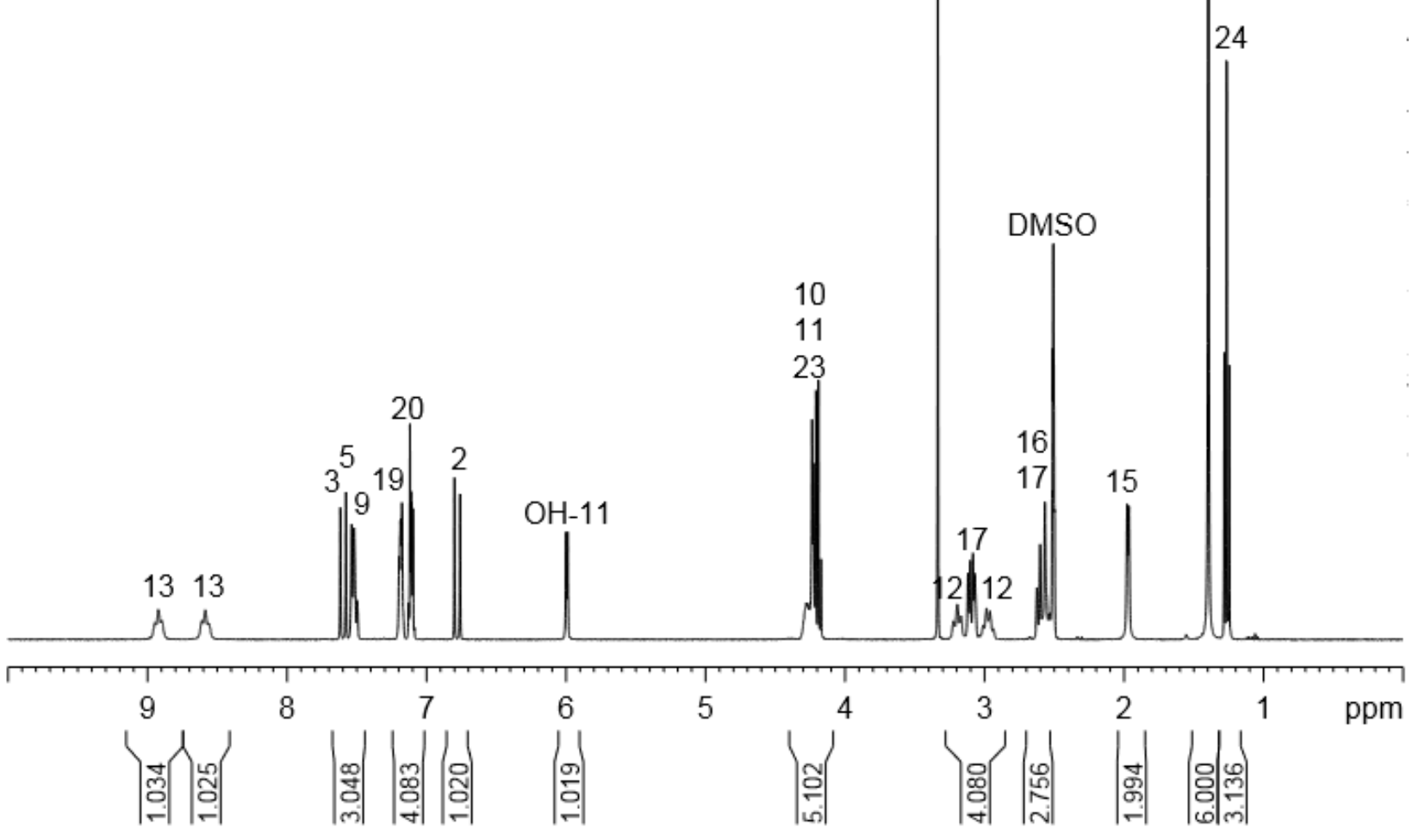




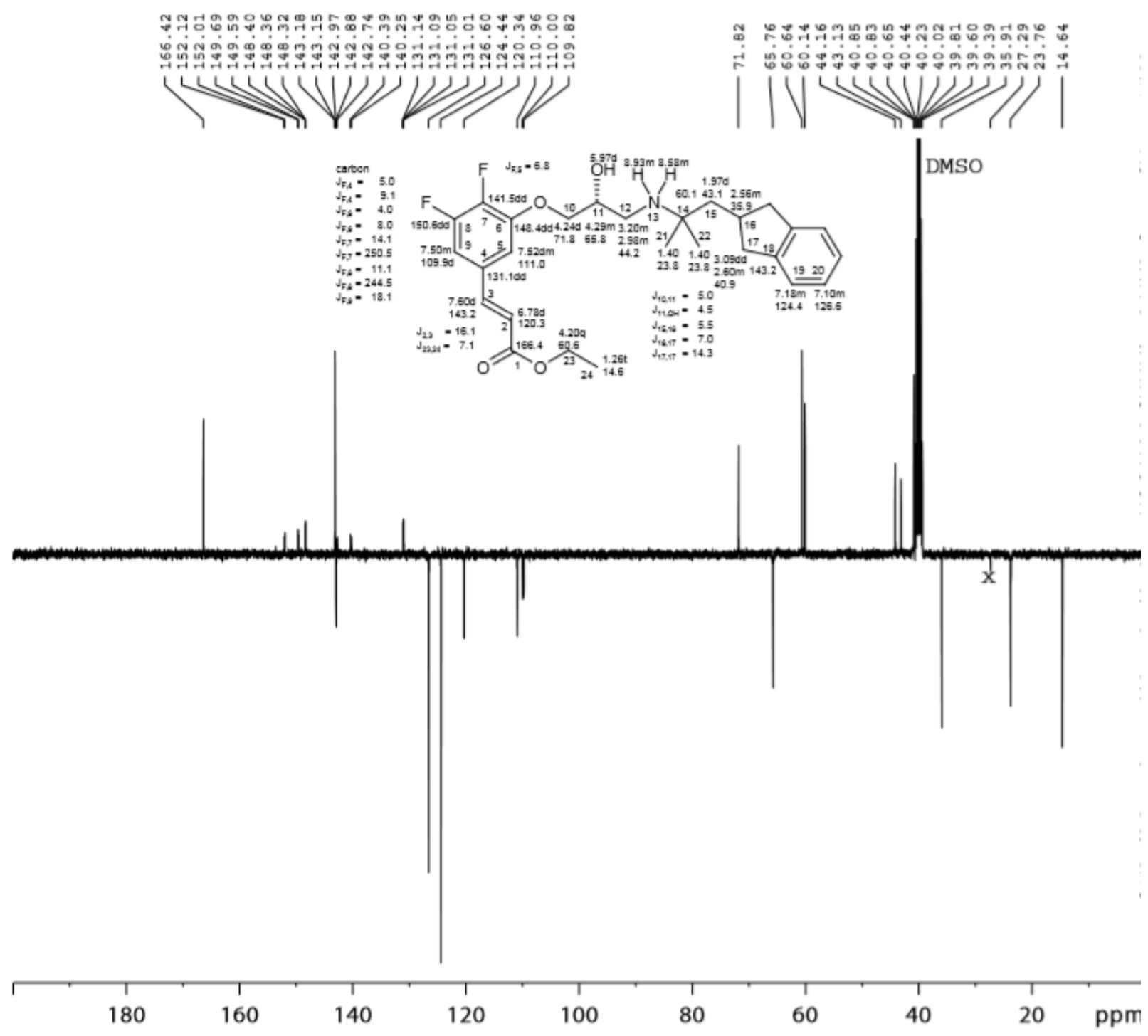



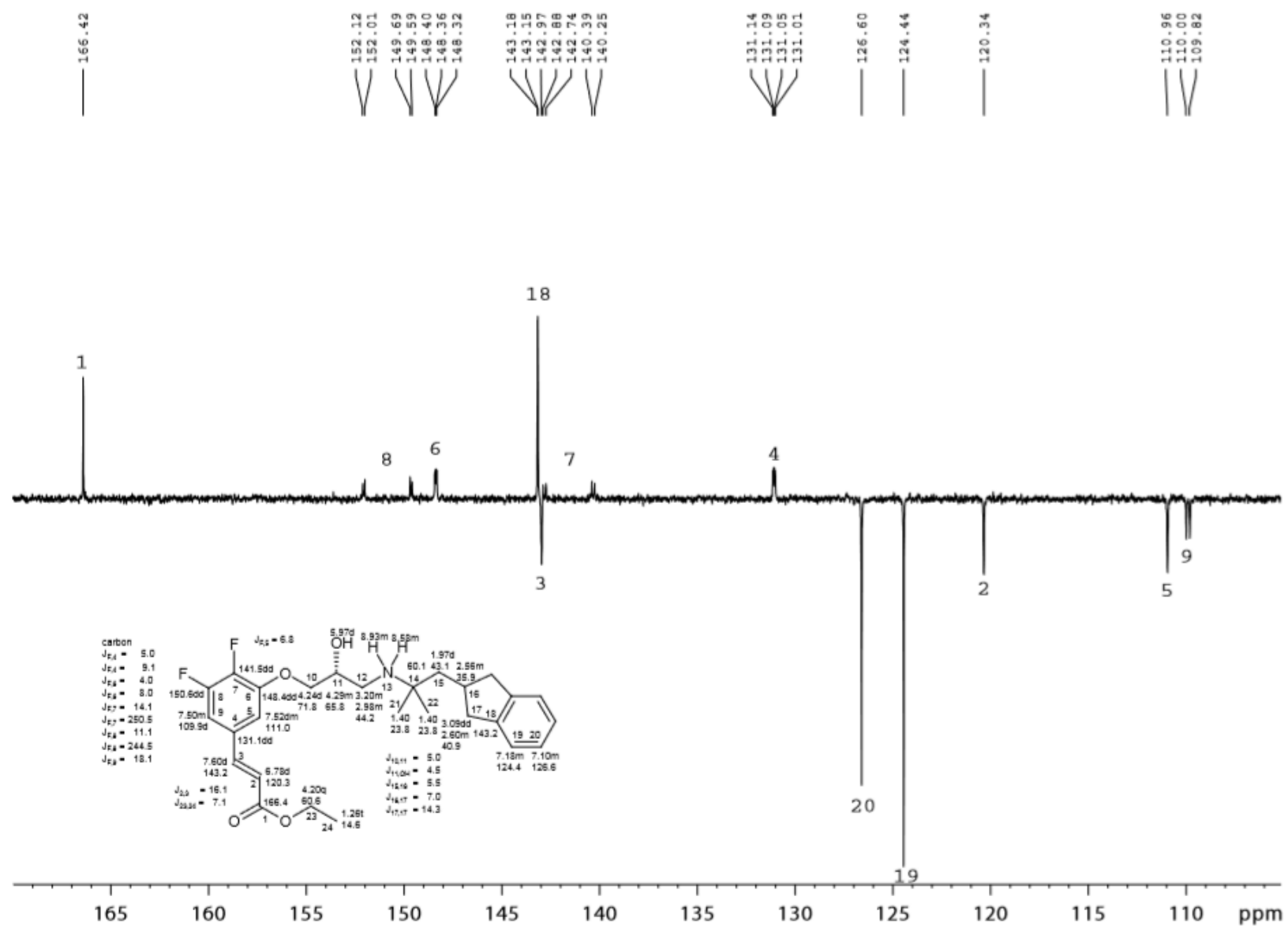


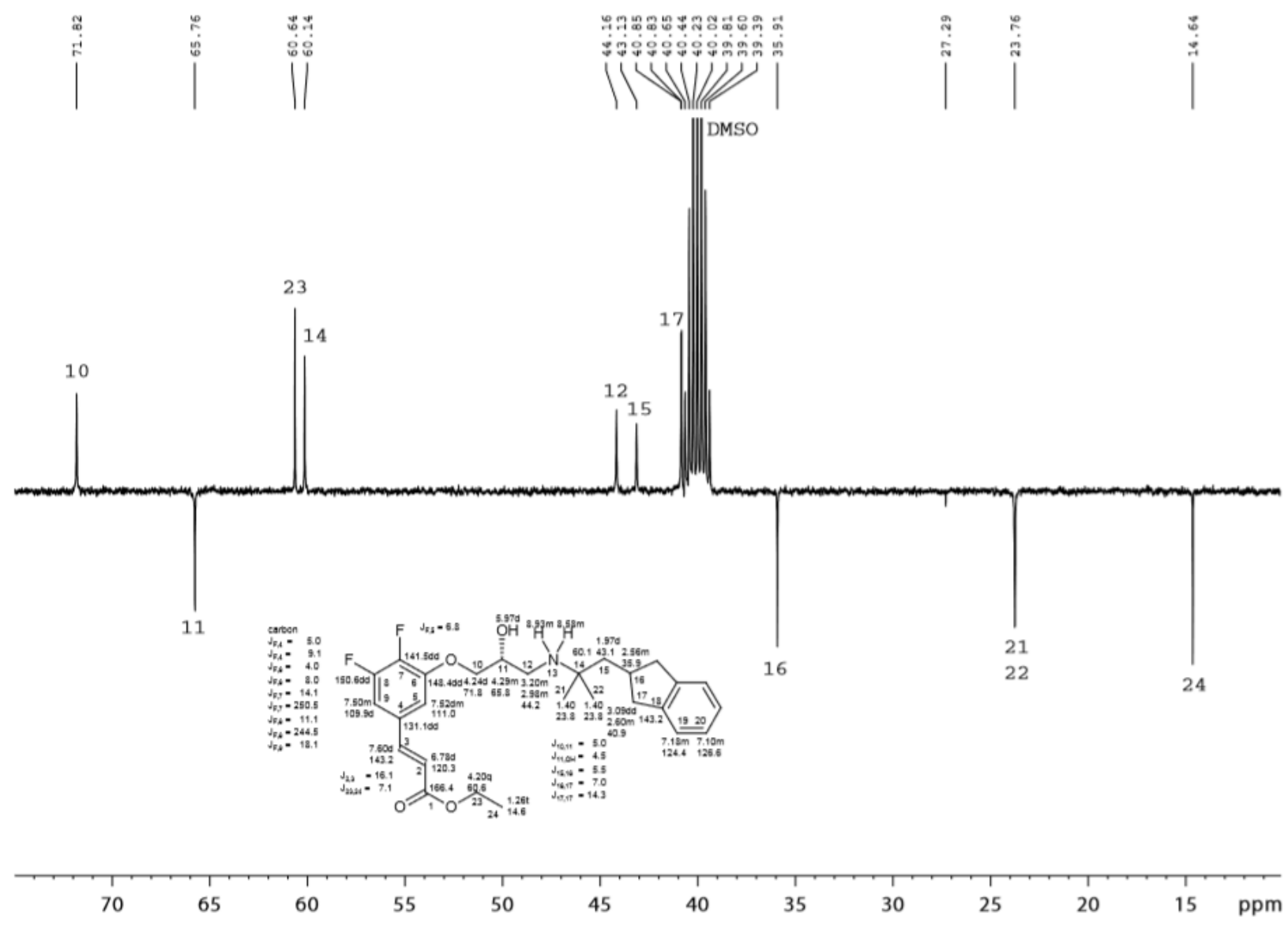


2. ${ }^{1} \mathrm{H}$ NMR monitoring of NMP-5-OOH generated by bubbling air through NMP at room temperature

Freshly opened NMP bottle:

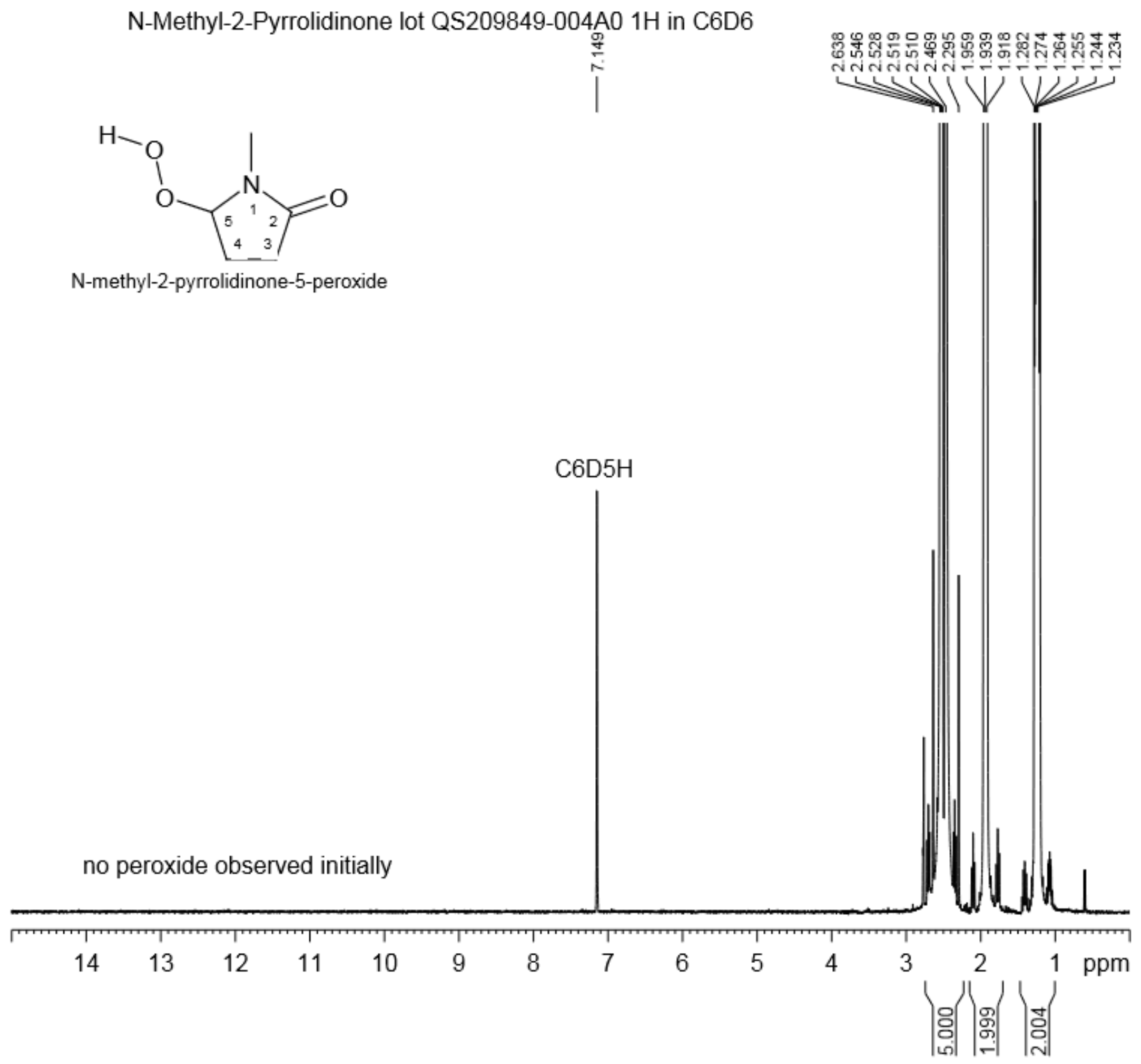


Day 1 after bubbling air through NMP at room temperature:

N-Methyl-2-Pyrrolidinone lot QS209849-004A1 (1 day air exposure) 1H in C6D6<smiles>CN1C(=O)C=CC1OO</smiles>

$\mathrm{N}$-methyl-2-pyrrolidinone-5-peroxide

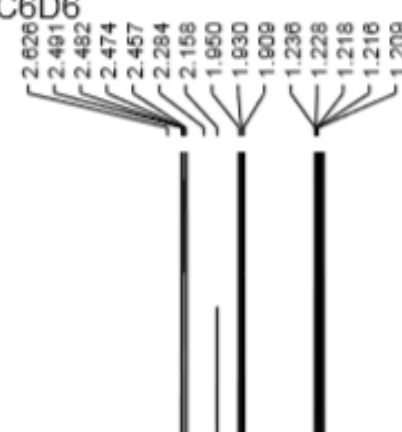

no peroxide observed

C6D5H

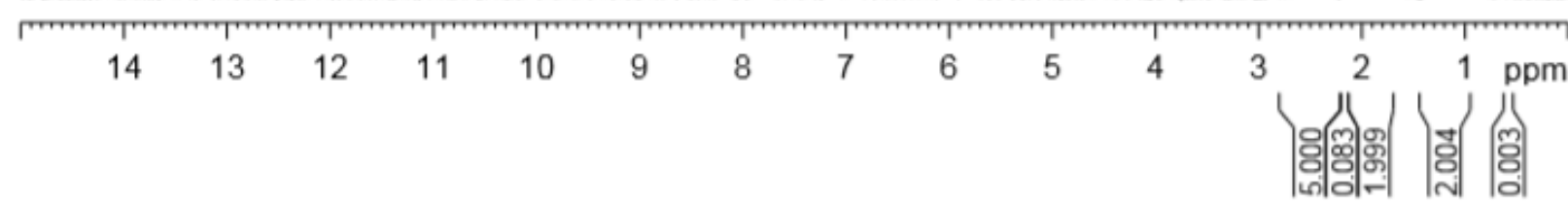


Day 5 after bubbling air through NMP at room temperature:

N-Methyl-2-Pyrrolidinone lot QS209849-004A2 1H in C6D6
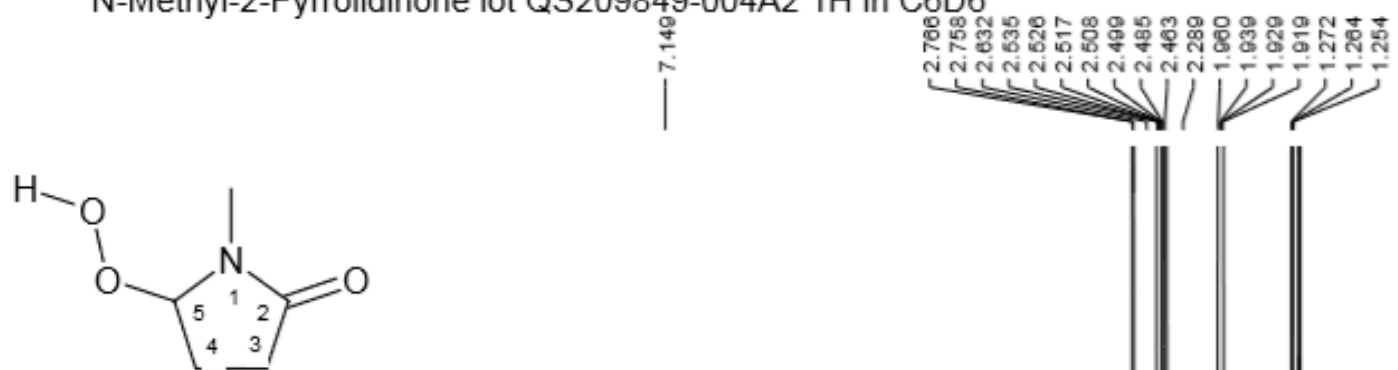

$\mathrm{N}$-methyl-2-pyrrolidinone-5-peroxide

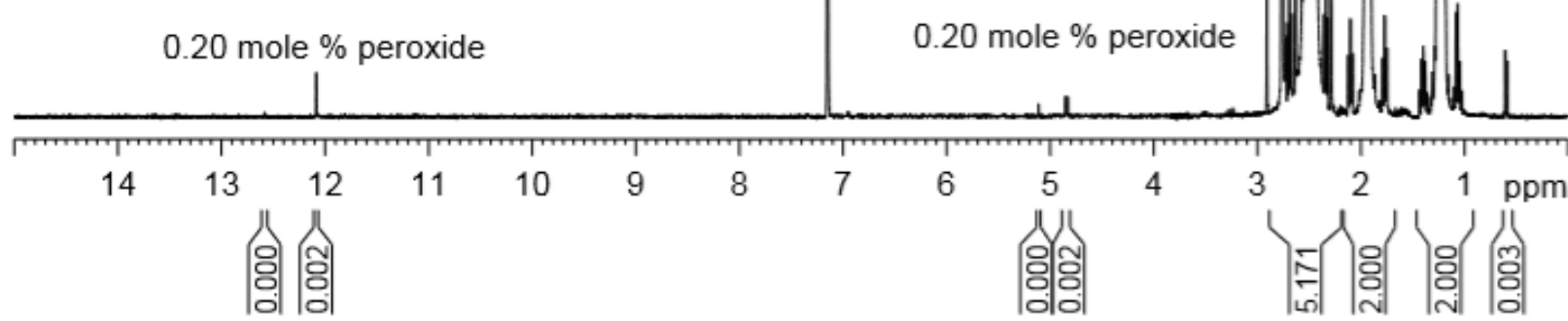


Day 9 after bubbling air through NMP at room temperature:

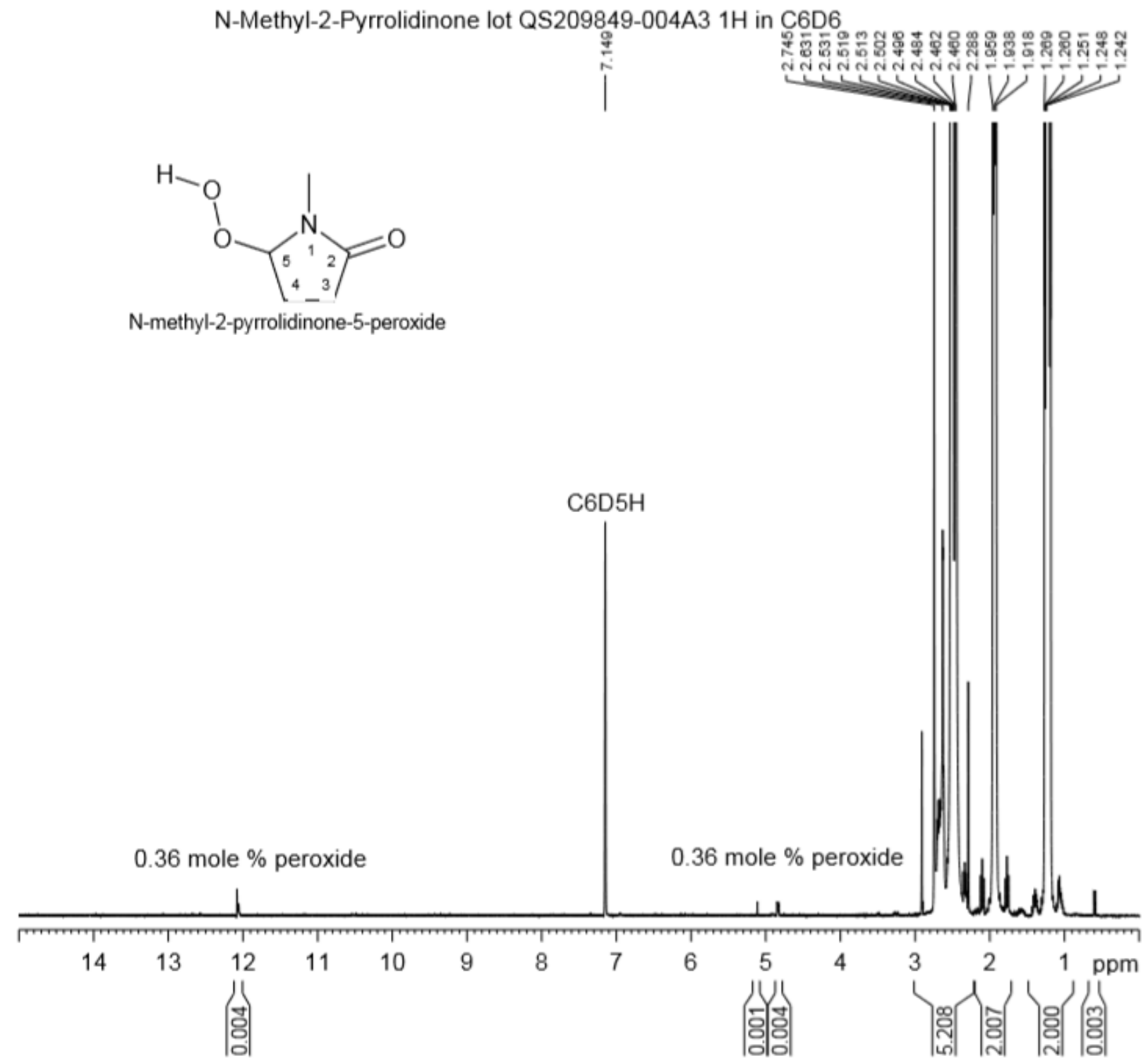


Day 13 after bubbling air through NMP at room temperature:

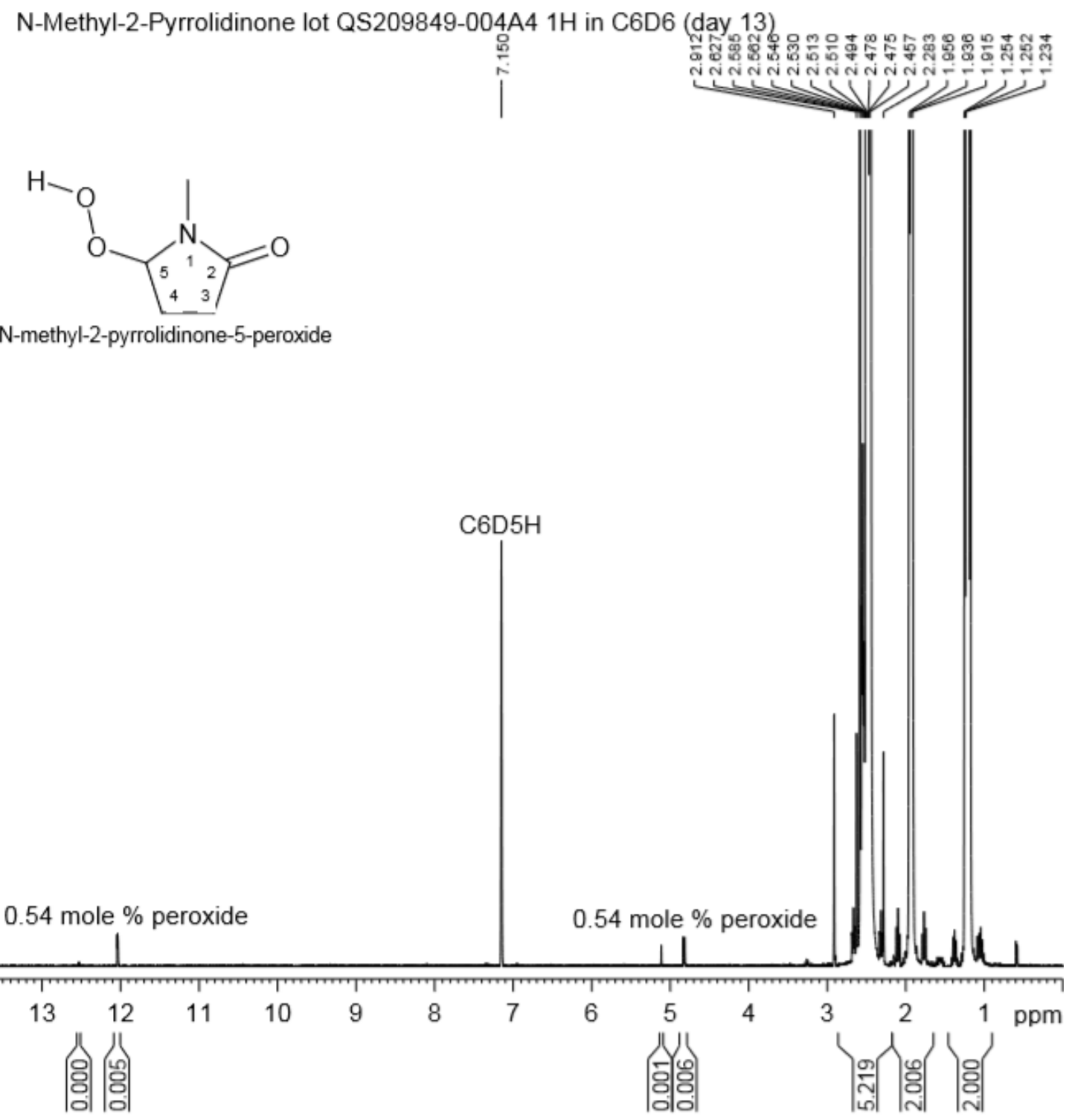


Day 19 after bubbling air through NMP at room temperature:

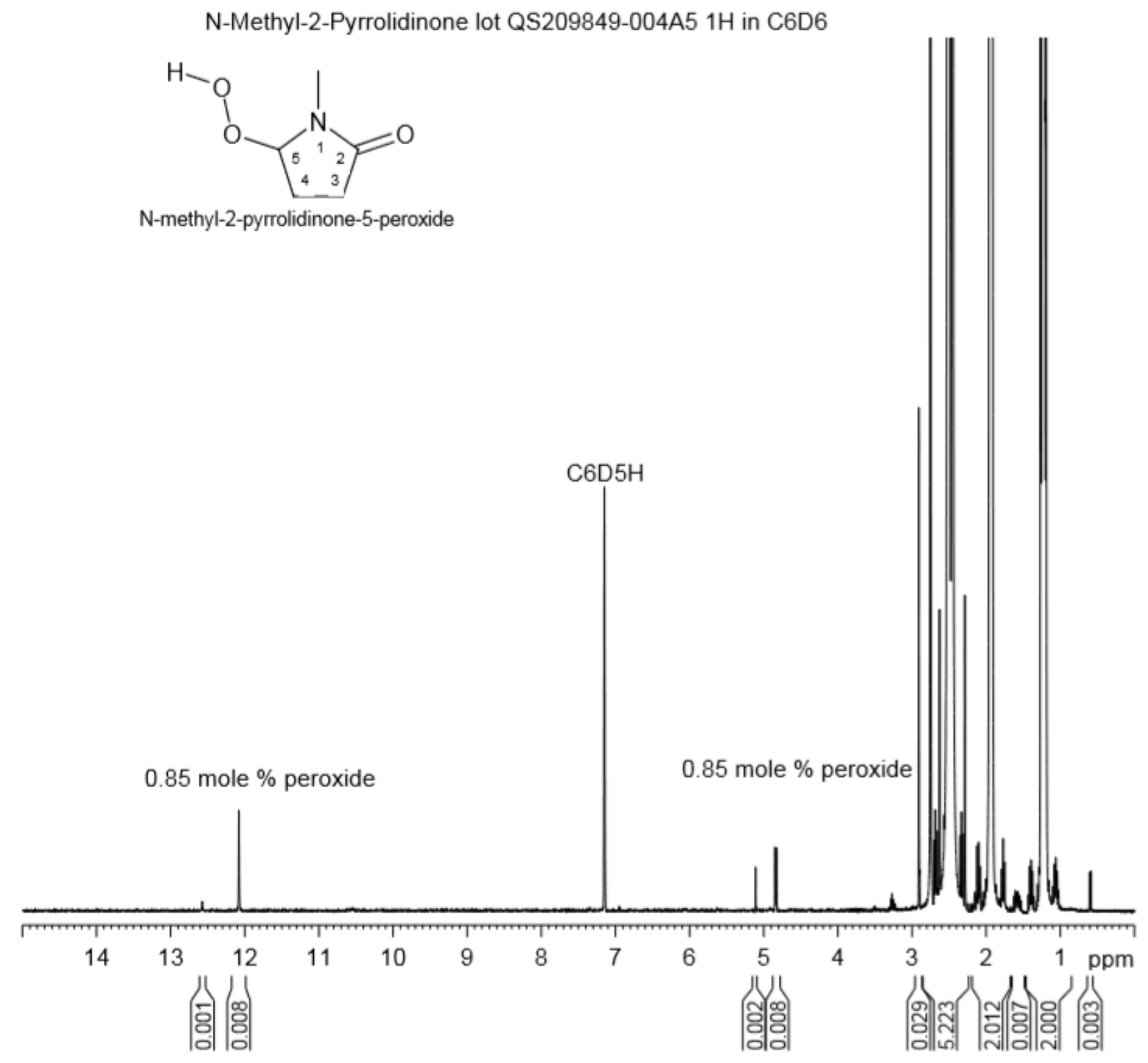


Day 26 after bubbling air through NMP at room temperature:

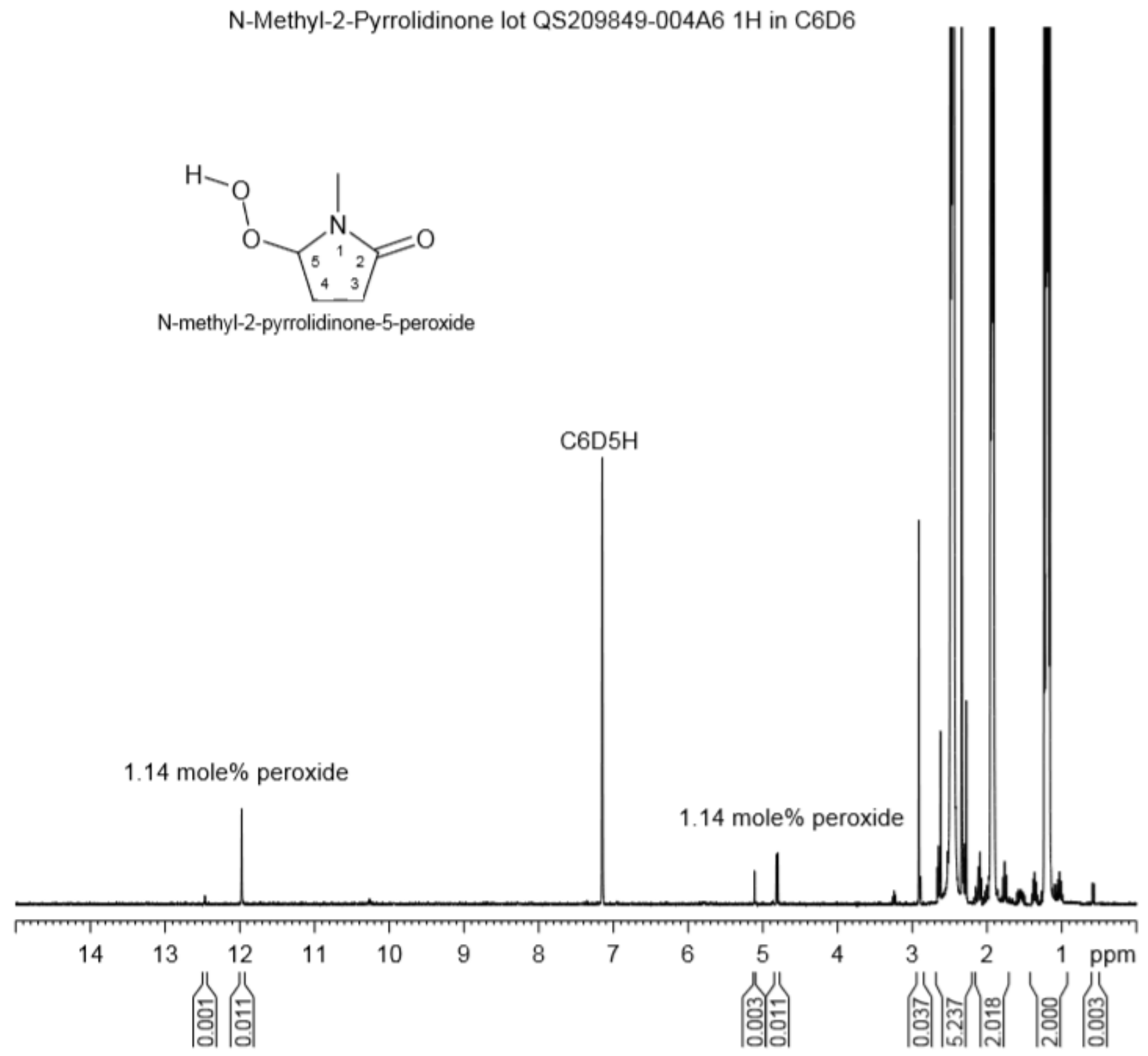


Day 33 after bubbling air through NMP at room temperature:

\section{N-Methyl-2-Pyrrolidinone lot QS209849-004A7 1H in C6D6}

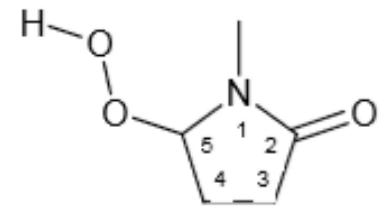

$\mathrm{N}$-methyl-2-pyrrolidinone-5-peroxide 
Day 44 after bubbling air through NMP at room temperature:

N-Methyl-2-Pyrrolidinone lot QS209849-004A8 1H in C6D6

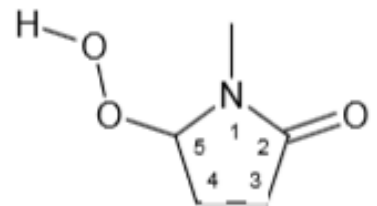

$\mathrm{N}$-methyl-2-pyrrolidinone-5-peroxide

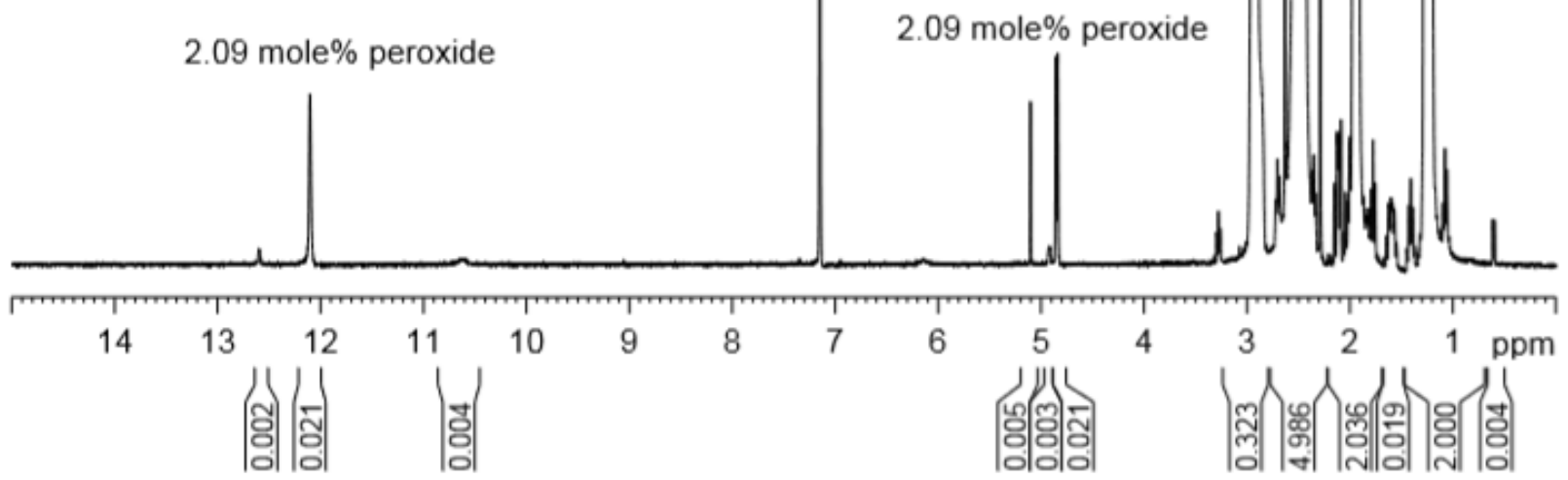


3. LCMS detection of NMP-5-OOH
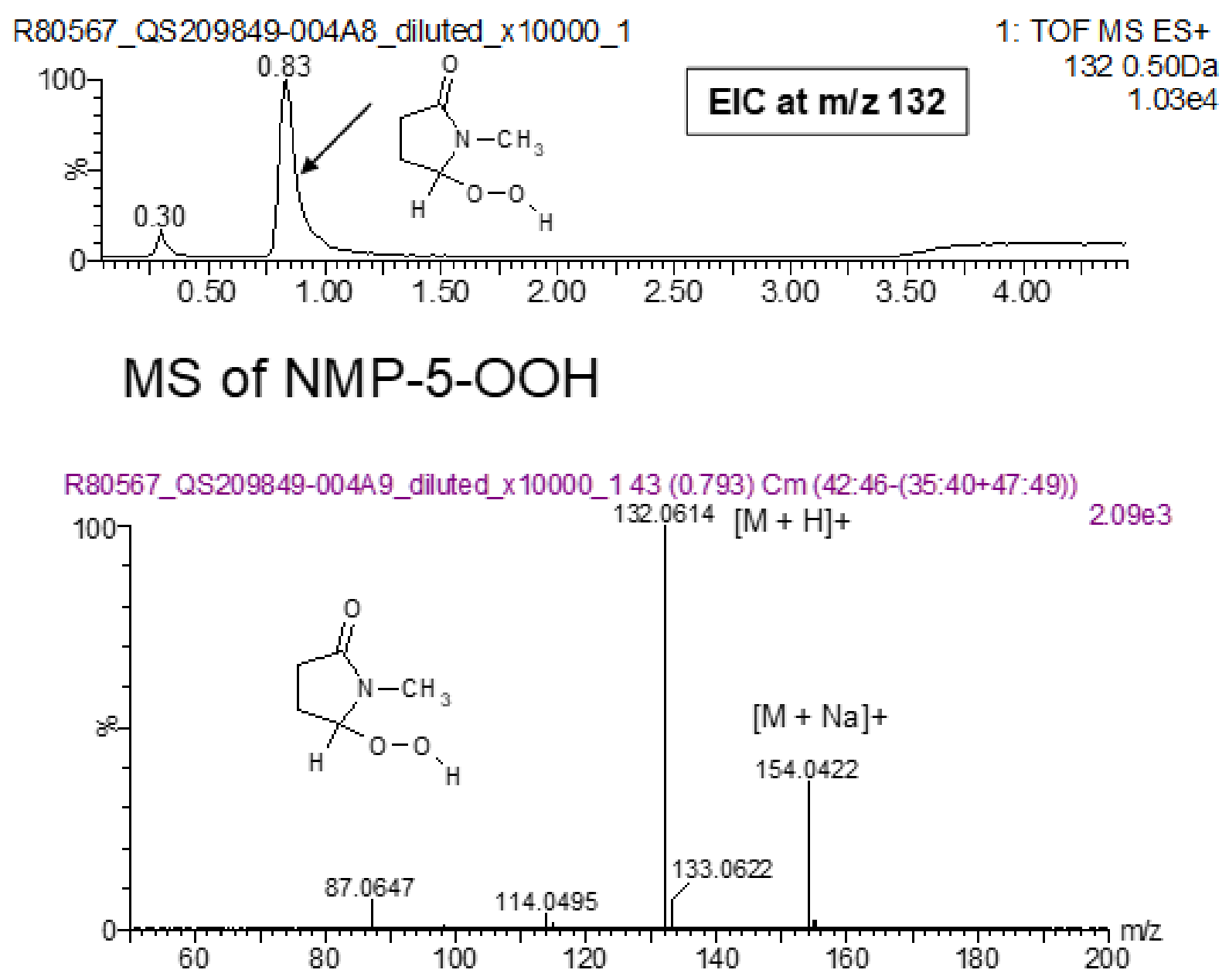